\title{
LOG CANONICAL MODELS FOR THE MODULI SPACE OF STABLE POINTED RATIONAL CURVES
}

\author{
HAN-BOM MOON \\ (Communicated by Lev Borisov)
}

\begin{abstract}
We run Mori's program for the moduli space of stable pointed rational curves with divisor $K+\sum a_{i} \psi_{i}$. We prove that the birational model for the pair is either the Hassett space of weighted pointed stable rational curves for the same weights or the GIT quotient of the product of projective lines with the linearization given by the same weights.
\end{abstract}

\section{INTRODUCTION}

The Knudsen-Mumford space $\bar{M}_{0, n}$, or the moduli space of stable pointed rational curves, is one of the most concrete and well-studied moduli spaces in algebraic geometry. For example, it is well-known that $\bar{M}_{0, n}$ is a smooth projective fine moduli space ([Kee92, Knu83]). Also the cohomology ring, the Chow ring, and the Picard group are known ( Kee92). There are several concrete constructions by using explicit methods such as smooth blow-ups (Kap93, Kee92]) or by geometric invariant theory (GIT) as quotients by $S L(2)$ ([HK00, KM11]). Furthermore, there are various different compactifications of the space of smooth pointed rational curves such as Hassett's moduli spaces of weighted stable pointed rational curves $\bar{M}_{0, \mathcal{A}}([\operatorname{Has} 03])$, the GIT quotients of the product of the projective lines (Kap93) with various effective linearizations, and the moduli spaces of pointed conics (GS10]). All of them are birational models of $\bar{M}_{0, n}$.

In spite of these numerous achievements, the birational geometric aspects of $\bar{M}_{0, n}$ are not fully understood yet. For instance, the Mori cone $\overline{N E}_{1}\left(\bar{M}_{0, n}\right)$ (dually, the nef cone $\left.\operatorname{Nef}\left(\bar{M}_{0, n}\right)\right)$ is unknown. There is only a conjectural description of this cone that is proved for $n \leq 7$ ([KMc96] $)$.

Conjecture 1.1 (F-conjecture). Any effective curve in $\bar{M}_{0, n}$ is numerically equivalent to a nonnegative linear combination of $F$-curves. In other words, every extremal ray of $\overline{N E}_{1}\left(\bar{M}_{0, n}\right)$ is generated by $F$-curve classes.

Recently, there has been a tremendous amount of interest in the birational geometry of $\bar{M}_{0, n}$ ([AS08, Fed10, FS08, GG11, GS10, GKM02, Has03, HK00, Kap93, Sim07]) and more generally of $\bar{M}_{g, n}$. In particular, one can run Mori's program (or the minimal model program) for $\bar{M}_{0, n}$ with a big $\mathbb{Q}$-divisor $D$ of $\bar{M}_{0, n}$ by finding a

Received by the editors October 3, 2011 and, in revised form, January 21, 2012. 2010 Mathematics Subject Classification. Primary 14D20, 14E30, 14H10. 
birational model

$$
\bar{M}_{0, n}(D):=\operatorname{Proj}\left(\bigoplus_{l \geq 0} H^{0}\left(\bar{M}_{0, n}, \mathcal{O}(l D)\right)\right),
$$

where the sum is taken over $l$ sufficiently divisible.

The most prominent two results in this direction are the following. Set $m=\left\lfloor\frac{n}{2}\right\rfloor$. Let $\epsilon_{k}$ be a rational number in the range $\frac{1}{m+1-k}<\epsilon_{k} \leq \frac{1}{m-k}$ for $k=1,2, \cdots, m-2$. For $\epsilon>0$, let $n \cdot \epsilon=(\epsilon, \cdots, \epsilon)$ be a symmetric weight datum.

Theorem 1.2 (Simpson [AS08, FS08, KM11, Sim07]). Let $\beta$ be a rational number satisfying $\frac{2}{n-1}<\beta \leq 1$ and let $D=\bar{M}_{0, n}-M_{0, n}$ denote the total boundary divisor. Then the log canonical model

$$
\bar{M}_{0, n}\left(K_{\bar{M}_{0, n}}+\beta D\right)=\operatorname{Proj}\left(\bigoplus_{l \geq 0} H^{0}\left(\bar{M}_{0, n}, \mathcal{O}\left(l\left(K_{\bar{M}_{0, n}}+\beta D\right)\right)\right)\right)
$$

satisfies the following:

(1) If $\frac{2}{m-k+2}<\beta \leq \frac{2}{m-k+1}$ for $1 \leq k \leq m-2$, then $\bar{M}_{0, n}\left(K_{\bar{M}_{0, n}}+\beta D\right) \cong$ $\bar{M}_{0, n \cdot \epsilon_{k}}$.

(2) If $\frac{2}{n-1}<\beta \leq \frac{2}{m+1}$, then $\bar{M}_{0, n}\left(K_{\bar{M}_{0, n}}+\beta D\right) \cong\left(\mathbb{P}^{1}\right)^{n} / / S L(2)$, where the quotient is taken with respect to the symmetric linearization $\mathcal{O}(1, \cdots, 1)$.

The other result concerning both nonsymmetric weights and higher genera is the following theorem of Fedorchuk. It is an answer to a question of Hassett ( Has03, Problem 7.1]).

Theorem 1.3 ([Fed10]). For every genus $g$ and weight datum $\mathcal{A}$, there exists a $\log$ canonical divisor $D_{g, \mathcal{A}}$ on $\bar{M}_{g, n}$ such that the log canonical model $\bar{M}_{g, n}\left(K_{\bar{M}_{g, n}}+\right.$ $\left.D_{g, \mathcal{A}}\right)$ is isomorphic to $\bar{M}_{g, \mathcal{A}}$.

In this paper, we prove a universal formula generalizing Theorem 1.2 to nonsymmetric weights $\mathcal{A}=\left(a_{1}, \cdots, a_{n}\right)$.

Theorem 1.4. Let $\mathcal{A}=\left(a_{1}, \cdots, a_{n}\right)$ be a weight datum. Then:

(1) If $\sum_{i=1}^{n} a_{i}>2$, then the log canonical model $\bar{M}_{0, n}\left(K_{\bar{M}_{0, n}}+\sum_{i=1}^{n} a_{i} \psi_{i}\right)$ is isomorphic to $\bar{M}_{0, \mathcal{A}}$.

(2) Assume that $n \geq 5$. If $\sum_{i=1}^{n} a_{i}=2$, then $\bar{M}_{0, n}\left(K_{\bar{M}_{0, n}}+\sum_{i=1}^{n} a_{i} \psi_{i}\right)$ is isomorphic to $\left(\mathbb{P}^{1}\right)^{n} / /{ }_{L} S L(2)$, where $L$ is the linearization $\mathcal{O}\left(a_{1}, \cdots, a_{n}\right)$.

In Theorem 1.4, item (2), if $n=4$ it is easy to check that $K_{\bar{M}_{0,4}}+\sum_{i=1}^{4} a_{i} \psi_{i}$ is numerically trivial.

Here is an outline of the proof of Theorem 1.4, item (1). Let $\Delta_{\mathcal{A}}=K_{\bar{M}_{0, n}}+$ $\sum_{i=1}^{n} a_{i} \psi_{i}$. Let $\varphi_{\mathcal{A}}: \bar{M}_{0, n} \rightarrow \bar{M}_{0, \mathcal{A}}$ be the reduction morphism (see Section 2.1). By computing the push-forwards and pull-backs of divisors (see Section 2.2), we prove that $\Delta_{\mathcal{A}}-\varphi_{\mathcal{A}}^{*} \varphi_{\mathcal{A} *}\left(\Delta_{\mathcal{A}}\right)$ is an effective divisor supported on the exceptional locus of $\varphi_{\mathcal{A}}$. Thus

$$
H^{0}\left(\bar{M}_{0, n}, \mathcal{O}\left(l \Delta_{\mathcal{A}}\right)\right) \cong H^{0}\left(\bar{M}_{0, \mathcal{A}}, \mathcal{O}\left(l \varphi_{\mathcal{A} *}\left(\Delta_{\mathcal{A}}\right)\right)\right)
$$


for any positive integer $l$ by [Deb01, Lemma 7.11]. Hence if we prove that $\varphi_{\mathcal{A} *}\left(\Delta_{\mathcal{A}}\right)$ is ample on $\bar{M}_{0, \mathcal{A}}$, then we have

$$
\begin{aligned}
\bar{M}_{0, n}\left(\Delta_{\mathcal{A}}\right) & =\operatorname{Proj}\left(\bigoplus_{l \geq 0} H^{0}\left(\bar{M}_{0, n}, \mathcal{O}\left(l \Delta_{\mathcal{A}}\right)\right)\right) \\
& \cong \operatorname{Proj}\left(\bigoplus_{l \geq 0} H^{0}\left(\bar{M}_{0, \mathcal{A}}, \mathcal{O}\left(l \varphi_{\mathcal{A} *}\left(\Delta_{\mathcal{A}}\right)\right)\right) \cong \bar{M}_{0, \mathcal{A}} .\right.
\end{aligned}
$$

For proving the ampleness of $\varphi_{\mathcal{A} *}\left(\Delta_{\mathcal{A}}\right)$, we follow the strategy of Fedorchuk in Fed10]. First, we can express $\varphi_{\mathcal{A} *}\left(\Delta_{\mathcal{A}}\right)$ in terms of tautological divisors on $\bar{M}_{0, \mathcal{A}}$. Then by using a positivity result of Fedorchuk (Proposition 3.3) and induction on the dimension, we prove that $\varphi_{\mathcal{A} *}\left(\Delta_{\mathcal{A}}\right)$ intersects all effective curves nonnegatively, so it is nef. Moreover, we prove that small perturbations of $\varphi_{\mathcal{A} *}\left(\Delta_{\mathcal{A}}\right)$ by boundary divisors are again nef. Since the Neron-Severi vector space $N^{1}\left(\bar{M}_{0, \mathcal{A}}\right)$ is generated by the boundary divisor classes, this implies that $\varphi_{\mathcal{A} *}\left(\Delta_{\mathcal{A}}\right)$ lies in the interior of $\operatorname{Nef}\left(\bar{M}_{0, \mathcal{A}}\right)$, so it is ample by Kleiman's criterion.

This paper is organized as follows. In section 2 we give some well-known facts about $\bar{M}_{0, \mathcal{A}}$ and its divisor classes. Essentially there is no new result in this section. In section 3 , we give a proof of Theorem 1.4 .

\section{SOME PRELIMINARIES}

2.1. Moduli space of weighted pointed rational stable curves. A weight datum $\mathcal{A}=\left(a_{1}, \cdots, a_{n}\right)$ is a sequence of rational numbers such that $0<a_{i} \leq 1$. A family of nodal curves of genus $g$ with $n$ marked points over a base scheme $B$ consists of a flat proper morphism $\pi: C \rightarrow B$ whose geometric fibers are nodal connected curves with arithmetic genus $g$, and $n$ sections $s_{1}, \cdots, s_{n}$ of $\pi$.

Definition 2.1 ([Has03, Section 2]). Let $\mathcal{A}$ be a weight datum satisfying $2 g-$ $2+\sum_{i=1}^{n} a_{i}>0$. A family of nodal curves of genus $g$ with $n$ marked points $\pi:\left(C, s_{1}, \cdots, s_{n}\right) \rightarrow B$ is $\mathcal{A}$-stable if

(1) the sections $s_{1}, \cdots, s_{n}$ lie in the smooth locus of $\pi$;

(2) for any subset $\left\{s_{i_{1}}, \cdots, s_{i_{r}}\right\}$ of nonempty intersection, $a_{i_{1}}+\cdots+a_{i_{r}} \leq 1$;

(3) $\omega_{\pi}+\sum_{i=1}^{n} a_{i} s_{i}$ is $\pi$-ample.

For any weight datum $\mathcal{A}$ such that $2 g-2+\sum_{i=1}^{n} a_{i}>0$, there exists a connected irreducible smooth Deligne-Mumford stack $\overline{\mathcal{M}}_{g, \mathcal{A}}$ such that its coarse moduli space $\bar{M}_{g, \mathcal{A}}$ is projective $\left(\left[\right.\right.$ Has03, Theorem 2.1]). Note that when $a_{1}=\cdots=a_{n}=1$, $\overline{\mathcal{M}}_{g, \mathcal{A}}=\overline{\mathcal{M}}_{g, n}$. If $g=0$, then $\overline{\mathcal{M}}_{0, \mathcal{A}}$ is a fine moduli space, so $\overline{\mathcal{M}}_{0, \mathcal{A}}=\bar{M}_{0, \mathcal{A}}$. From now on, we will focus on the $g=0$ case only.

Let $\mathcal{A}=\left(a_{1}, \cdots, a_{n}\right), \mathcal{B}=\left(b_{1}, \cdots, b_{n}\right)$ be two weight data and suppose that $a_{i} \geq b_{i}$ for all $i=1,2, \cdots, n$. Then there exists a birational reduction morphism ([Has03, Theorem 4.1])

$$
\varphi_{\mathcal{A}, \mathcal{B}}: \bar{M}_{0, \mathcal{A}} \rightarrow \bar{M}_{0, \mathcal{B}}
$$

For $\left(C, s_{1}, \cdots, s_{n}\right) \in \bar{M}_{0, \mathcal{A}}, \varphi_{\mathcal{A}, \mathcal{B}}\left(C, s_{1}, \cdots, s_{n}\right)$ is obtained by collapsing components on which $\omega_{C}+\sum b_{i} s_{i}$ fails to be ample. Every reduction morphism is a 
composition of smooth blow-downs ([KM11, Mo11]). In this article, we use reduction morphisms from $\bar{M}_{0, n}$ only. So we use the more concise notation

$$
\varphi_{\mathcal{A}}:=\varphi_{(1, \cdots, 1), \mathcal{A}}: \bar{M}_{0, n} \rightarrow \bar{M}_{0, \mathcal{A}} .
$$

2.2. Tautological divisors on $\bar{M}_{0, \mathcal{A}}$. In this section, we recall some information about several functorial divisors on $\bar{M}_{0, \mathcal{A}}$. The results in this section are drawn from several sources, including [AC96, AC99, Fed10, FS08, HM98, Has03.

Let $[n]=\{1, \cdots, n\}$. For $I \subset[n]$ such that $2 \leq|I| \leq n-2$, let $D_{I} \subset \bar{M}_{0, n}$ be the closure of the locus of curves $C$ with two irreducible components $C_{I}, C_{I^{c}}$ such that the $i$-th marked point is lying on $C_{I}$ if and only if $i \in I$. So $D_{I}=D_{I^{c}}$. These divisors are called boundary divisors. By Kee92, boundary divisors generate the Picard group $\operatorname{Pic}\left(\bar{M}_{0, n}\right)$ and the Neron-Severi vector space $N^{1}\left(\bar{M}_{0, n}\right)$.

Definition 2.2. Let $\mathcal{A}=\left(a_{1}, \cdots, a_{n}\right)$ be a weight datum such that $\sum_{i=1}^{n} a_{i}>2$. For $I \subset[n]$, let $w_{I}:=\sum_{i \in I} a_{i}$. There are two kinds of boundary divisor classes in $\bar{M}_{0, \mathcal{A}}$ for a general weight datum $\mathcal{A}$.

(1) Boundary of nodal curves: Suppose that $w_{I^{c}} \geq w_{I}>1$. Let $D_{I}$ be the divisor of $\bar{M}_{0, \mathcal{A}}$ corresponding to the closure of the locus of curves with two irreducible components $C_{I}, C_{I^{c}}$ and $s_{i} \in C_{I}$ if and only if $i \in I$. Let $D_{\text {nod }}$ be the sum of all boundaries of nodal curves.

(2) Boundary of curves with coincident sections: Suppose that $I=\{i, j\}$ and $w_{I} \leq 1$. Since $w=w_{[n]}>2$, this implies $w_{I^{c}}>w_{I}$. Let $D_{I}$ be the locus of $s_{i}=s_{j}$. Let $D_{\mathrm{sec}}$ be the sum of all boundaries of curves with coincident sections.

Since the reduction morphism $\varphi_{\mathcal{A}}$ is a composition of smooth blow-ups, one can easily derive the following push-forward and pull-back formulas for divisor classes.

Lemma 2.3. Let $\varphi_{\mathcal{A}}: \bar{M}_{0, n} \rightarrow \bar{M}_{0, \mathcal{A}}$ be the reduction morphism. For $I \subset[n]$, let $w_{I}=\sum_{i \in I} a_{i}$. Assume $w_{I} \leq w_{I^{c}}$ for every $D_{I}$. Then:

(1) $\varphi_{\mathcal{A} *}\left(D_{I}\right)= \begin{cases}0, & |I| \geq 3 \text { and } w_{I} \leq 1, \\ D_{I}, & \text { otherwise. }\end{cases}$

(2) $\varphi_{\mathcal{A}}^{*}\left(D_{I}\right)= \begin{cases}D_{I}+\sum_{J \supset I, w_{J} \leq 1} D_{J}, & D_{I} \text { is a boundary of curves } \\ & \text { with coincident sections, } \\ D_{I}, & \text { otherwise. }\end{cases}$

Let $\pi: U \rightarrow \bar{M}_{0, \mathcal{A}}$ be the universal curve and $\sigma_{i}: \bar{M}_{0, \mathcal{A}} \rightarrow U$ for $i=1, \cdots, n$ be the universal sections. Let $\omega=\omega_{U / \bar{M}_{0, \mathcal{A}}}$ be the relative dualizing bundle. We define tautological divisors on $\bar{M}_{0, \mathcal{A}}$ as follows:

(1) The kappa class is $\kappa=\pi_{*}\left(c_{1}^{2}(\omega)\right)$. This definition is different from $\kappa_{1}$ in AC99.

(2) For $1 \leq i \leq n$, let $\mathbb{L}_{i}$ be the line bundle on $\bar{M}_{0, \mathcal{A}}$ whose fiber over $\left(C, s_{1}, s_{2}, \cdots, s_{n}\right)$ is $\left.\Omega_{C}\right|_{s_{i}}$, a cotangent space at $s_{i}$ in $C$. The $i$-th psi class is $\psi_{i}=c_{1}\left(\mathbb{L}_{i}\right)$. In terms of intersection theory, $\psi_{i}=\pi_{*}\left(\omega \cdot \sigma_{i}\right)=\pi_{*}\left(-\sigma_{i}^{2}\right)$. The total psi class is $\psi=\sum_{i=1}^{n} \psi_{i}$.

(3) The boundary of curves with coincident sections $D_{\{i, j\}}$ is equal to $\pi_{*}\left(\sigma_{i} \cdot \sigma_{j}\right)$. 
We focus on the genus zero case only, so the lambda class $\lambda=c_{1}\left(\pi_{*}(\omega)\right)$ is zero.

Next, consider the push-forwards and pull-backs of several divisors.

Lemma 2.4. Let $\varphi_{\mathcal{A}}: \bar{M}_{0, n} \rightarrow \bar{M}_{0, \mathcal{A}}$ be the reduction morphism. Then:

(1) $\varphi_{\mathcal{A} *}\left(K_{\bar{M}_{0, n}}\right)=K_{\bar{M}_{0, \mathcal{A}}}$.

(2) $\varphi_{\mathcal{A} *}\left(\psi_{i}\right)=\psi_{i}+\sum_{\substack{j \neq i \\ a_{i}+a_{j}<1}} D_{\{i, j\}}$.

(3) $\varphi_{\mathcal{A}}^{*}\left(\psi_{i}\right)=\psi_{i}-\sum_{\substack{i \in I \\ w_{I} \leq 1}}^{a_{i}+D_{I}}$.

Proof. Since the discrepancy is supported on the exceptional locus, item (1) follows immediately. Items (2) and (3) are more careful observations of the proof of [FS08. Lemma 2.4] and [FS08, Lemma 2.8] respectively. Item (3) is also a corollary of cumbersome computation using Lemmas 2.3 and 2.9 .

For $I=\left\{i_{1}, \cdots, i_{r}\right\} \subset[n]$, let $D_{I}$ be a boundary of nodal curves. Set $I^{c}=$ $\left\{j_{1}, \cdots, j_{s}\right\}$. Then $D_{I}$ is isomorphic to $\bar{M}_{0, \mathcal{A}_{I}} \times \bar{M}_{0, \mathcal{A}_{I}}$, where $\mathcal{A}_{I}=\left(a_{i_{1}}, \cdots, a_{i_{r}}, 1\right)$ and $\mathcal{A}_{I^{c}}=\left(a_{j_{1}}, \cdots, a_{j_{s}}, 1\right)$. Let $\eta_{I}: \bar{M}_{0, \mathcal{A}_{I}} \times \bar{M}_{0, \mathcal{A}_{I^{c}}} \rightarrow D_{I} \hookrightarrow \bar{M}_{0, \mathcal{A}}$ be the inclusion morphism. Define $\pi_{i}$ for $i=1,2$ as the projection from $\bar{M}_{0, \mathcal{A}_{I}} \times \bar{M}_{0, \mathcal{A}_{I^{c}}}$ to the $i$-th component.

Lemma 2.5. Let $\eta_{I}: \bar{M}_{0, \mathcal{A}_{I}} \times \bar{M}_{0, \mathcal{A}_{I^{c}}} \rightarrow D_{I} \hookrightarrow \bar{M}_{0, \mathcal{A}}$ be the inclusion morphism. Let $p$ (resp. q) denote the last index of $\mathcal{A}_{I}$ (resp. $\mathcal{A}_{I^{c}}$ ) with weight one. Then:

(1) $\eta_{I}^{*}(\kappa)=\pi_{1}^{*}\left(\kappa+\psi_{p}\right)+\pi_{2}^{*}\left(\kappa+\psi_{q}\right)$.

(2) $\eta_{I}^{*}\left(\psi_{i}\right)= \begin{cases}\pi_{1}^{*}\left(\psi_{i}\right), & i \in I, \\ \pi_{2}^{*}\left(\psi_{i}\right), & i \in I^{c} .\end{cases}$

(3) For $J \subset[n]$, suppose that $D_{J}$ is a boundary of nodal curves:

$$
\eta_{I}^{*}\left(D_{J}\right)= \begin{cases}\pi_{1}^{*}\left(D_{J}\right), & J \subsetneq I, \\ \pi_{2}^{*}\left(D_{J}\right), & J \subsetneq I^{c}, \\ \pi_{1}^{*}\left(-\psi_{p}\right)+\pi_{2}^{*}\left(-\psi_{q}\right), & J=I, \\ 0, & \text { otherwise. }\end{cases}
$$

(4) Suppose that $a_{i}+a_{j} \leq 1$ :

$$
\eta_{I}^{*}\left(D_{\{i, j\}}\right)= \begin{cases}\pi_{1}^{*}\left(D_{\{i, j\}}\right), & i, j \in I, \\ \pi_{2}^{*}\left(D_{\{i, j\}}\right), & i, j \in I^{c} \\ 0, & \text { otherwise. }\end{cases}
$$

Proof. The proofs of these items are essentially identical to the case of $\bar{M}_{0, n}$. Item (1) is in AC96, Section 1]. Items (2) and (4) are clear. The only nonobvious part of item (3) is due to [HM98, Proposition 3.31].

Let $J \subset[n]$ be a subset of $[n]$ such that $\sum_{j \in J} a_{j} \leq 1$. Let $\mathcal{A}^{\prime}$ be the new weight datum obtained by replacing weights indexed by $J$ by one weight $\sum_{j \in J} a_{j}$. Then the locus of $\sigma_{i}=\sigma_{j}$ for all $i, j \in J$ is isomorphic to $\bar{M}_{0, \mathcal{A}^{\prime}}$ because we can replace sections $\left\{\sigma_{j}\right\}_{j \in J}$ by one section with weight $\sum_{j \in J} a_{j}$. Let $\chi_{J}: \bar{M}_{0, \mathcal{A}^{\prime}} \hookrightarrow \bar{M}_{0, \mathcal{A}}$ be the replacement morphism.

Lemma 2.6. Let $\chi_{J}: \bar{M}_{0, \mathcal{A}^{\prime}} \rightarrow \bar{M}_{0, \mathcal{A}}$ be the replacement morphism. Let $p$ denote the unique index of $\mathcal{A}^{\prime}$ replacing indices in $J$. Then:

(1) $\chi_{J}^{*}\left(\psi_{i}\right)= \begin{cases}\psi_{i}, & i \notin J, \\ \psi_{p}, & i \in J .\end{cases}$ 
(2) $\chi_{J}^{*}\left(D_{\text {nod }}\right)=D_{\text {nod }}$.

(3) Suppose that $D_{\{i, j\}}$ is a boundary of curves with coincident sections:

$$
\chi_{J}^{*}\left(D_{\{i, j\}}\right)= \begin{cases}D_{\{i, j\}}, & i, j \notin J, \\ D_{\{i, p\}}, & i \notin J, j \in J, \\ -\psi_{p}, & i, j \in J .\end{cases}
$$

Proof. Essentially this is a restatement of [FS08, Lemma 2.9].

Finally, let us recall the canonical divisor of $\bar{M}_{0, \mathcal{A}}$. The following formula is a consequence of Hassett's computation of the canonical divisor and the weighted version of Mumford's relation. By [Has03, Section 3.3.1],

$$
K_{\bar{M}_{0, \mathcal{A}}}=\frac{13}{12} \kappa-\frac{11}{12} D_{\text {nod }}+\sum_{i=1}^{n} \psi_{i} .
$$

In the proof of Mumford's relation $\kappa=12 \lambda-D_{\text {nod }}$ ([Mum77]) for $\bar{M}_{g}$, Mumford used only the facts 1 ) the parametrized curves have at worst nodal singularities only, 2) the singular locus of the morphism from the universal curve to the moduli space has codimension two. Thus the same proof holds for $\bar{M}_{0, \mathcal{A}}$, too. Note that $\lambda=0$ for the genus zero case.

\section{Lemma 2.7.}

$$
K_{\bar{M}_{0, \mathcal{A}}}=-2 D_{\mathrm{nod}}+\sum_{i=1}^{n} \psi_{i}=2 \kappa+\sum_{i=1}^{n} \psi_{i} .
$$

2.3. Numerical results for $\bar{M}_{0, n}$. It is well known that the Neron-Severi vector space $N^{1}\left(\bar{M}_{0, n}\right)$ of numerical divisor classes is generated by boundary divisors ( Kee92 $)$. Many natural divisors on $\bar{M}_{0, n}$ are already expressed as linear combinations of boundary divisors. For $j=2,3, \cdots, n-2$, let $D_{j}=\sum_{|I|=j} D_{I}$.

Lemma 2.8 ([ Pan97, Proposition 2]).

$$
K_{\bar{M}_{0, n}} \equiv \sum_{j=2}^{\lfloor n / 2\rfloor}\left(\frac{j(n-j)}{n-1}-2\right) D_{j} .
$$

To describe psi-classes as combinations of boundary divisors, we recall a notation in [FG03, Section 2]. For $I \subset[n]$, let

$$
D_{j}^{I, i}:=\sum_{\substack{A \subset I,|A|=i \\ B \subset I^{c},|B|=j}} D_{A \cup B}
$$

Lemma 2.9. On $\bar{M}_{0, n}$,

(1)

$$
\psi_{i} \equiv \sum_{j=1}^{n-3} \frac{(n-1-j)(n-2-j)}{(n-1)(n-2)} D_{j}^{\{i\}, 1}
$$

$$
\psi \equiv \sum_{j=2}^{\lfloor n / 2\rfloor}\left(\frac{j(n-j)}{n-1}\right) D_{j} \equiv K_{\bar{M}_{0, n}}+2 D
$$


Proof. The first item is [FG03, Lemma 1]. The second item follows from a direct computation using (11) and Lemma 2.8

Let $I_{1} \sqcup I_{2} \sqcup I_{3} \sqcup I_{4}=[n]$ be a partition. Let $F_{I_{1}, I_{2}, I_{3}, I_{4}}$ be an F-curve class corresponding to the partition ([KMc96, Section 4]). The intersection numbers of F-curves and boundary divisors are well-known.

Lemma 2.10 ([KMc96, Lemma 4.3]). Let $F=F_{I_{1}, I_{2}, I_{3}, I_{4}}$ be an F-curve, and let $D_{I}$ be a boundary divisor. Then:

(1) $D_{I} \cdot F=-1$ if $I$ or $I^{c}$ is one of $I_{i}$.

(2) $D_{I} \cdot F=1$ if $I$ is $I_{i} \sqcup I_{j}$ for some distinct $i, j$.

(3) Otherwise, $D_{I} \cdot F=0$.

From these results, we can calculate all the intersection numbers we want.

\section{Proof of the MAIN THEOREM}

In this section, we prove our main theorem. Throughout this section, we will assume $n \geq 4$. If $n=3$, then $\bar{M}_{0,3}$ is a point, so there is nothing to prove.

Theorem 3.1. Let $\mathcal{A}=\left(a_{1}, \cdots, a_{n}\right)$ be a weight datum such that $\sum_{i=1}^{n} a_{i}>2$. Then the log canonical model $\bar{M}_{0, n}\left(K_{\bar{M}_{0, n}}+\sum_{i=1}^{n} a_{i} \psi_{i}\right)$ is isomorphic to $\bar{M}_{0, \mathcal{A}}$.

Proof. Fix a weight datum $\mathcal{A}=\left(a_{1}, \cdots, a_{n}\right)$. Let $\Delta_{\mathcal{A}}=K_{\bar{M}_{0, n}}+\sum_{i=1}^{n} a_{i} \psi_{i}$. Set $T=\left\{I \subset[n]\left|w_{I}=\sum_{i \in I} a_{i} \leq 1,2 \leq\right| I \mid \leq n-2\right\}$. By Lemmas 2.4 and 2.7] it is straightforward to check that

$$
\begin{aligned}
\varphi_{\mathcal{A} *}\left(\Delta_{\mathcal{A}}\right) & =K_{\bar{M}_{0, \mathcal{A}}}+\sum_{i=1}^{n} a_{i} \psi_{i}+\sum_{\substack{i<j \\
a_{i}+a_{j} \leq 1}}\left(a_{i}+a_{j}\right) D_{\{i, j\}} \\
& =-2 D_{\text {nod }}+\sum_{i=1}^{n}\left(1+a_{i}\right) \psi_{i}+\sum_{\substack{i<j \\
a_{i}+a_{j} \leq 1}}\left(a_{i}+a_{j}\right) D_{\{i, j\}} .
\end{aligned}
$$

By Lemmas 2.3 and 2.4

$$
\begin{aligned}
\varphi_{\mathcal{A}}^{*} \varphi_{\mathcal{A} *}\left(\Delta_{\mathcal{A}}\right)= & -2 D_{\text {nod }}+2 \sum_{I \in T} D_{I}+\sum_{i=1}^{n}\left(1+a_{i}\right) \psi_{i}-\sum_{I \in T}\left(|I|+w_{I}\right) D_{I} \\
& +\sum_{\substack{i<j \\
a_{i}+a_{j} \leq 1}}\left(a_{i}+a_{j}\right) D_{\{i, j\}}+\sum_{\substack{I \in T \\
|I| \geq 3}}(|I|-1) w_{I} D_{I} \\
= & -2 D_{\text {nod }}+\sum_{i=1}^{n}\left(1+a_{i}\right) \psi_{i}+\sum_{I \in T}(|I|-2)\left(w_{I}-1\right) D_{I} .
\end{aligned}
$$

So

$$
\Delta_{\mathcal{A}}-\varphi_{\mathcal{A}}^{*} \varphi_{\mathcal{A} *}\left(\Delta_{\mathcal{A}}\right)=\sum_{I \in T}(|I|-2)\left(1-w_{I}\right) D_{I} .
$$

Note that for every $I \in T,|I| \geq 2$ and $w_{I} \leq 1$ by the definition of $T$. So the difference $\Delta_{\mathcal{A}}-\varphi_{\mathcal{A}}^{*} \varphi_{\mathcal{A} *}\left(\Delta_{\mathcal{A}}\right)$ is supported on the exceptional locus of $\varphi_{\mathcal{A}}$ and is effective. This implies that

$$
H^{0}\left(\bar{M}_{0, n}, \Delta_{\mathcal{A}}\right) \cong H^{0}\left(\bar{M}_{0, n}, \varphi_{\mathcal{A}}^{*} \varphi_{\mathcal{A} *}\left(\Delta_{\mathcal{A}}\right)\right) \cong H^{0}\left(\bar{M}_{0, \mathcal{A}}, \varphi_{\mathcal{A} *}\left(\Delta_{\mathcal{A}}\right)\right)
$$


by [Deb01, Lemma 7.11]. The same statement holds for a positive multiple of $\Delta_{\mathcal{A}}$, too. Therefore from the definition of the log canonical model, we obtain

$$
\begin{aligned}
\bar{M}_{0, n}\left(K_{\bar{M}_{0, n}}+\sum_{i=1}^{n} a_{i} \psi_{i}\right) & =\operatorname{Proj}\left(\bigoplus_{l \geq 0} H^{0}\left(\bar{M}_{0, n}, \mathcal{O}\left(l \Delta_{\mathcal{A}}\right)\right)\right) \\
& =\operatorname{Proj}\left(\bigoplus_{l \geq 0} H^{0}\left(\bar{M}_{0, \mathcal{A}}, \mathcal{O}\left(l \varphi_{\mathcal{A} *}\left(\Delta_{\mathcal{A}}\right)\right)\right)\right) .
\end{aligned}
$$

If we prove that $\varphi_{\mathcal{A} *}\left(\Delta_{\mathcal{A}}\right)$ is ample, then the last birational model is exactly $\bar{M}_{0, \mathcal{A}}$. So to prove the main theorem, it suffices to show that $\varphi_{\mathcal{A} *}\left(\Delta_{\mathcal{A}}\right)$ is ample on $\bar{M}_{0, \mathcal{A}}$. This is done in Propositions 3.2 and 3.5 .

Proposition 3.2. Let $\mathcal{A}=\left(a_{1}, \cdots, a_{n}\right)$ be a weight datum and let $\Delta_{\mathcal{A}}=K_{\bar{M}_{0, n}}+$ $\sum_{i=1}^{n} a_{i} \psi_{i}$. Then $\varphi_{\mathcal{A} *}\left(\Delta_{\mathcal{A}}\right)$ is a nef divisor on $\bar{M}_{0, \mathcal{A}}$.

The key ingredient is the following positivity result of Fedorchuk ([Fed10]). Fedorchuk gives an elementary and beautiful intersection theoretical proof of this result. As Fedorchuk mentioned in [Fed10, it can be proved by using the semipositivity method of Kollár in [Kol90, Corollary 4.6, Proposition 4.7].

Proposition 3.3 ([Fed10, Proposition 2.1]). Let $\pi: S \rightarrow B$ be a generically smooth family of nodal curves of arithmetic genus $g$, with $n$ sections $\sigma_{1}, \cdots, \sigma_{n}$ over a smooth complete curve $B$. For a weight datum $\mathcal{A}=\left(a_{1}, \cdots, a_{n}\right)$, suppose that

$$
L:=\omega_{\pi}+\sum_{i=1}^{n} a_{i} \sigma_{i}
$$

is $\pi$-nef. Suppose further that $\sigma_{i_{1}}, \cdots, \sigma_{i_{k}}$ can coincide only if $\sum_{j=1}^{k} a_{i_{j}} \leq 1$. Then $L$ is nef on $S$.

If $\pi: S \rightarrow B$ is a generically smooth family of $\mathcal{A}$-stable curves or, more generally, $\mathcal{A}$-semistable curves (allowing irreducible rational components with 2 nodes and no marked points), then the hypotheses of Proposition 3.3 are satisfied by the definition of $\mathcal{A}$-stability.

We need an effectivity result first.

Lemma 3.4. Let $\pi: S \rightarrow B$ be a family of $\mathcal{A}$-semi-stable rational curves with $n$ sections $\sigma_{1}, \cdots, \sigma_{n}$ over a smooth complete curve $B$. Then $2 \omega_{\pi}+\sum_{i=1}^{n} \sigma_{i}$ is effective.

Proof. We will use induction on $n$. For the $n=4$ case, the result is a direct computation.

By [HM98, Section 3.C], $S$ has at worst $A_{k}$ singularities only. An $A_{k}$ singularity is Du Val, so if $\rho: \tilde{S} \rightarrow S$ is a minimal resolution, then $\omega_{\pi \circ \rho}=\rho^{*}\left(\omega_{\pi}\right)$ and $\rho_{*}\left(\omega_{\pi \circ \rho}\right)=\omega_{\rho}$. Thus we may assume that $S$ is smooth.

Suppose that for $J \subset[n]$ with $|J| \geq 2, \sigma_{i}=\sigma_{j}$ for all $i, j \in J$. We may assume that $J=\{1,2, \cdots, m\}$ for some $m \leq n$. After pulling-back along $\chi_{J}$ : $\bar{M}_{0, \mathcal{A}^{\prime}} \rightarrow \bar{M}_{0, \mathcal{A}}$ (see Section [2.2), we may assume that $\left(\pi: S \rightarrow B, \sigma_{1}, \cdots, \sigma_{n}\right)$ is a family of $\mathcal{A}^{\prime}$-stable curves $\left(\pi: S \rightarrow B, \sigma_{m}, \sigma_{m+1}, \cdots, \sigma_{n}\right)$ with $|J|-1$ additional sections $\sigma_{1}, \sigma_{2}, \cdots, \sigma_{m-1}$. By the induction hypothesis, $2 \omega_{\pi}+\sum_{i=m}^{n} \sigma_{i}$ is effective. 
So $2 \omega_{\pi}+\sum_{i=1}^{n} \sigma_{i}=\left(2 \omega_{\pi}+\sum_{i=m}^{n} \sigma_{i}\right)+\sum_{i=1}^{m-1} \sigma_{i}$ is effective, too. Thus we may assume that all sections are distinct.

After taking several blow-ups along points where two or more sections meet, we obtain a family of $(1, \cdots, 1)$-semi-stable curves $\left(\pi_{1}: S_{1} \rightarrow B, \sigma_{1}^{1}, \cdots, \sigma_{n}^{1}\right)$.

Let $\rho_{1}: S_{1} \rightarrow S$ be the blow-up. If there exist $(-1)$ curves with exactly 2 sections, after contracting these $(-1)$ curves by blowing-down, we get a family $\left(\pi_{2}: S_{2} \rightarrow B, \sigma_{1}^{2}, \cdots, \sigma_{n}^{2}\right)$ of $(1 / 2, \cdots, 1 / 2)$-semi-stable curves. Let $\rho_{2}: S_{1} \rightarrow S_{2}$ be the blow-down morphism. Over $S_{2}, 2 \omega_{\pi_{2}}+\sum_{i=1}^{n} \sigma_{i}^{2}$ is nef by Proposition 3.3 and thus effective.

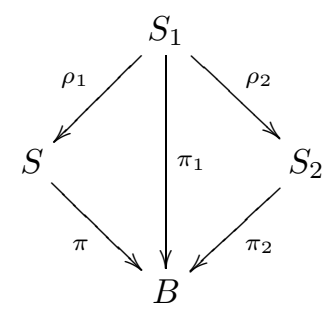

From $(1 / 2, \cdots, 1 / 2)$-stability, we know that for each point in $S_{2}$, at most two sections meet at that point. Let $x_{1}, \cdots, x_{k} \in S_{2}$ be points with coincident sections. Then $\rho_{2}$ is the blow-up along $x_{1}, \cdots, x_{k}$. Let $E_{1}, \cdots, E_{k}$ be the exceptional divisors. By the blow-up formula, $\omega_{\pi_{1}}=\rho_{2}^{*}\left(\omega_{\pi_{2}}\right)+\sum_{j=1}^{k} E_{j}$. Also $\sum_{i=1}^{n} \sigma_{i}^{1}=\rho_{2}^{*}\left(\sum_{i=1}^{n} \sigma_{i}^{2}\right)-$ $2 \sum_{j=1}^{k} E_{j}$. Thus

$$
2 \omega_{\pi_{1}}+\sum_{i=1}^{n} \sigma_{i}^{1}=\rho_{2}^{*}\left(2 \omega_{\pi_{2}}+\sum_{i=1}^{n} \sigma_{i}^{2}\right),
$$

so $2 \omega_{\pi_{1}}+\sum_{i=1}^{n} \sigma_{i}^{1}$ is effective.

Finally, $\rho_{1 *}\left(\omega_{\pi_{1}}\right)=\omega_{\pi}$ and $\rho_{1 *}\left(\sigma_{i}^{1}\right)=\sigma_{i}$ since $\rho_{1}$ is a composition of point blowups. Thus $2 \omega_{\pi}+\sum_{i=1}^{n} \sigma_{i}=\rho_{1 *}\left(2 \omega_{\pi_{1}}+\sum_{i=1}^{n} \sigma_{i}^{1}\right)$ is a push-forward of an effective divisor. Hence it is effective, too.

Proof of Proposition 3.2 . For the $n=4$ case, since $\bar{M}_{0, \mathcal{A}} \cong \bar{M}_{0, n} \cong \mathbb{P}^{1}, K_{\bar{M}_{0, n}} \equiv$ $\mathcal{O}(-2)$ and $\psi_{i} \equiv \mathcal{O}(1)$, the result is a consequence of a simple direct computation. So we can use induction on the number $n$ of marked points.

To prove the nefness of $\varphi_{\mathcal{A} *}\left(\Delta_{\mathcal{A}}\right)$, it suffices to show that for every complete irreducible curve $B \rightarrow \bar{M}_{0, \mathcal{A}}$, the restriction of $\left.\varphi_{\mathcal{A} *}\left(\Delta_{\mathcal{A}}\right)\right|_{B}$ has nonnegative degree. By composing with the normalization $B^{\nu} \rightarrow B$, we may assume that $B$ is smooth.

By equations (4) and (5), it is straightforward to check that

$$
\begin{aligned}
\varphi_{\mathcal{A} *}\left(\Delta_{\mathcal{A}}\right) & =2 \kappa+\sum_{i=1}^{n}\left(1+a_{i}\right) \psi_{i}+\sum_{\substack{i<j \\
a_{i}+a_{j} \leq 1}}\left(a_{i}+a_{j}\right) D_{\{i, j\}} \\
& =\pi_{*}\left(2 \omega^{2}+\sum_{i=1}^{n}\left(1+a_{i}\right)\left(\omega \cdot \sigma_{i}\right)+\sum_{\substack{i<j \\
a_{i}+a_{j} \leq 1}}\left(a_{i}+a_{j}\right)\left(\sigma_{i} \cdot \sigma_{j}\right)\right) .
\end{aligned}
$$

For a boundary divisor $D_{I}$ of nodal curves, let $\eta_{I}: \bar{M}_{0, \mathcal{A}_{I}} \times \bar{M}_{0, \mathcal{A}_{I^{c}}} \rightarrow D_{I} \hookrightarrow \bar{M}_{0, \mathcal{A}}$ be the inclusion of boundary. We will use the same notation as in Section 2.2. By 
Lemma 2.5 and (11), it is straightforward to check

$$
\eta_{I}^{*}\left(\varphi_{\mathcal{A} *}\left(\Delta_{\mathcal{A}}\right)\right)=\pi_{1}^{*}\left(\varphi_{\mathcal{A}_{I^{*}}}\left(\Delta_{\mathcal{A}_{I}}\right)\right)+\pi_{2}^{*}\left(\varphi_{\mathcal{A}_{I^{c}} *}\left(\Delta_{\mathcal{A}_{I^{c}}}\right)\right) .
$$

Thus for a curve $B$ supported on a boundary of nodal curves, the degree of $\varphi_{\mathcal{A} *}\left(\Delta_{\mathcal{A}}\right)$ is nonnegative by induction. Therefore it suffices to check for a family $S \rightarrow B$ of nodal curves over a smooth curve $B$, whose general fiber is a nonsingular curve.

Note that $\omega \cdot \sigma_{i}=-\sigma_{i}^{2}$ by adjunction and $\sigma_{i} \cdot \sigma_{j}=0$ if $a_{i}+a_{j}>1$. Therefore

$$
\begin{aligned}
& 2 \omega^{2}+\sum_{i=1}^{n}\left(1+a_{i}\right)\left(\omega \cdot \sigma_{i}\right)+\sum_{\substack{i<j \\
a_{i}+a_{j} \leq 1}}\left(a_{i}+a_{j}\right)\left(\sigma_{i} \cdot \sigma_{j}\right) \\
= & 2 \omega^{2}+\sum_{i=1}^{n}\left(\omega \cdot \sigma_{i}\right)+\sum_{i=1}^{n} 2 a_{i}\left(\omega \cdot \sigma_{i}\right)+\sum_{i=1}^{n} a_{i} \sigma_{i}^{2}+\sum_{i<j}\left(a_{i}+a_{j}\right)\left(\sigma_{i} \cdot \sigma_{j}\right) \\
= & \left(\omega+\sum_{i=1}^{n} a_{i} \sigma_{i}\right) \cdot\left(2 \omega+\sum_{i=1}^{n} \sigma_{i}\right) .
\end{aligned}
$$

Hence it suffices to check that $\left.\operatorname{deg} \pi_{*}\left(\left(\omega+\sum_{i=1}^{n} a_{i} \sigma_{i}\right) \cdot\left(2 \omega+\sum_{i=1}^{n} \sigma_{i}\right)\right)\right|_{B} \geq 0$. By Proposition 3.3. $\omega+\sum_{i=1}^{n} a_{i} \sigma_{i}$ is nef on $S$. By Lemma 3.4, $2 \omega+\sum_{i=1}^{n} \sigma_{i}$ is effective on $S$. Thus the intersection is nonnegative and the result follows.

Next, we prove the ampleness of $\varphi_{\mathcal{A} *}\left(\Delta_{\mathcal{A}}\right)$. This is an application of the perturbation technique of Fedorchuk and Smyth introduced in [FS08].

Proposition 3.5. With the same hypotheses of Proposition $3.2, \varphi_{\mathcal{A} *}\left(\Delta_{\mathcal{A}}\right)$ is an ample divisor on $\bar{M}_{0, \mathcal{A}}$.

Proof. Fix a metric $\|\cdot\|_{\mathcal{A}}$ on $N^{1}\left(\bar{M}_{0, \mathcal{A}}\right)$ for each weight datum $\mathcal{A}$. We will prove the following statement: For $\bar{M}_{0, \mathcal{A}}$, there exists $\epsilon_{\mathcal{A}}>0$ such that $\varphi_{\mathcal{A} *}\left(\Delta_{\mathcal{A}}\right)+P$ is nef for every $P \in N^{1}\left(\bar{M}_{0, \mathcal{A}}\right)$ satisfying $\|P\|_{\mathcal{A}}<\epsilon_{\mathcal{A}}$. This implies that $\varphi_{\mathcal{A} *}\left(\Delta_{\mathcal{A}}\right)$ lies in the interior of $\operatorname{Nef}\left(\bar{M}_{0, \mathcal{A}}\right)$, so by Kleiman's criterion, $\varphi_{\mathcal{A} *}\left(\Delta_{\mathcal{A}}\right)$ is ample.

We will use induction on $n$. When $n=4$, then $\bar{M}_{0, \mathcal{A}} \cong \mathbb{P}^{1}$ and the result is straightforward.

Let $B$ be an integral complete curve on $\bar{M}_{0, \mathcal{A}}$. Since we consider only the intersection numbers, we may assume $B$ is nonsingular by applying normalization. We will divide it into three cases:

Case $1 . B$ is in a component of nodal boundary.

By (13) and induction hypothesis, when we restrict $\varphi_{\mathcal{A} *}\left(\Delta_{\mathcal{A}}\right)$ to a component of the boundary of nodal curves, the restriction is ample. So there exists $\epsilon_{\mathcal{A}, I}>0$ such that $\eta_{I}^{*}\left(\varphi_{\mathcal{A} *}\left(\Delta_{\mathcal{A}}\right)+P\right)$ is nef for all $P \in N^{1}\left(\bar{M}_{0, \mathcal{A}}\right)$ such that $\|P\|_{\mathcal{A}}<\epsilon_{\mathcal{A}, I}$.

Case 2. A general point of $B$ parametrizes a smooth curve, and there exists $J \subset[n]$ with $|J| \geq 2$ such that $\sigma_{i}=\sigma_{j}$ for all $i, j \in J$.

We may assume that $J$ is maximal among such subsets. In this case, $B$ is contained in the image of $\chi_{J}: \bar{M}_{0, \mathcal{A}^{\prime}} \rightarrow \bar{M}_{0, \mathcal{A}}$ defined in Section 2.2 . Let $p$ be the 
unique index of $\mathcal{A}^{\prime}=\left(a_{j}^{\prime}\right)$ replacing indices in $J$. Then by (5) and Lemma 2.6.

$$
\begin{aligned}
& \chi_{J}^{*}\left(\varphi_{\mathcal{A} *}\left(\Delta_{\mathcal{A}}\right)\right)=\chi_{J}^{*}\left(-2 D_{\text {nod }}+\sum_{i=1}^{n}\left(1+a_{i}\right) \psi_{i}+\sum_{\substack{i<j \\
a_{i}+a_{j} \leq 1}}\left(a_{i}+a_{j}\right) D_{\{i, j\}}\right) \\
& =-2 D_{\mathrm{nod}}+\sum_{i \in J^{c}}\left(1+a_{i}\right) \psi_{i}+\sum_{i \in J}\left(1+a_{i}\right) \psi_{p}+\sum_{\substack{i<j, i, j \in J^{c} \\
a_{i}+a_{j} \leq 1}}\left(a_{i}+a_{j}\right) D_{\{i, j\}} \\
& +\sum_{\substack{i \in J, j \in J^{c} \\
\sum_{i \in J} a_{i}+a_{j} \leq 1}}\left(a_{i}+a_{j}\right) D_{\{p, j\}}-\sum_{i<j, i, j \in J}\left(a_{i}+a_{j}\right) \psi_{p} \\
& =-2 D_{\text {nod }}+\sum_{i \in J^{c}}\left(1+a_{i}\right) \psi_{i}+\left(1+\sum_{j \in J} a_{j}\right) \psi_{p}+(|J|-1) \psi_{p}+\sum_{\substack{i<j, i, j \in J^{c} \\
a_{i}+a_{j} \leq 1}}\left(a_{i}+a_{j}\right) D_{\{i, j\}} \\
& +\sum_{j \in J^{c}}\left(\left(\sum_{i \in J} a_{i}\right)+a_{j}\right) D_{\{p, j\}}+(|J|-1) \sum_{j \in J^{c}} a_{j} D_{\{p, j\}}-(|J|-1)\left(\sum_{i \in J} a_{i}\right) \psi_{p} \\
& \sum_{i \in J} a_{i}+a_{j} \leq 1 \quad \sum_{i \in J} a_{i}+a_{j} \leq 1 \\
& =\varphi_{\mathcal{A}^{\prime} *}\left(\Delta_{\mathcal{A}^{\prime}}\right)+(|J|-1)\left(\left(1-\sum_{i \in J} a_{i}\right) \psi_{p}+\sum_{\substack{j \in J^{c} \\
\sum_{i \in J} a_{i}+a_{j} \leq 1}} a_{j} D_{\{p, j\}}\right) \\
& =\varphi_{\mathcal{A}^{\prime *}}\left(\Delta_{\mathcal{A}^{\prime}}\right)+(|J|-1) \pi_{*}\left(\left(\omega+\sum a_{j}^{\prime} \sigma_{j}\right) \cdot \sigma_{p}\right) .
\end{aligned}
$$

The index of the first sum in the third line follows from the fact that $D_{\{i, j\}}$ for $i \in J, j \notin J$ meets $\chi_{J}\left(\bar{M}_{0, \mathcal{A}^{\prime}}\right)$ only if $\sum_{i \in J} a_{i}+a_{j} \leq 1$.

By induction hypothesis, $\varphi_{\mathcal{A}^{\prime} *}\left(\Delta_{\mathcal{A}^{\prime}}\right)$ is ample. Since $\omega+\sum a_{j}^{\prime} \sigma_{j}$ is nef by Proposition 3.2 the second term in the last line of (15) is nonnegative on $B$ (see also [Fed10, Theorem 1]). Hence there exists $\epsilon_{\mathcal{A}, J}>0$ such that $\chi_{J}^{*}\left(\varphi_{\mathcal{A} *}\left(\Delta_{\mathcal{A}}\right)+P\right.$ ) is nef for all $P \in N^{1}\left(\bar{M}_{0, \mathcal{A}}\right)$ with $\|P\|_{\mathcal{A}}<\epsilon_{\mathcal{A}, J}$.

Case 3. Otherwise.

In this case, a general point of $B$ parametrizes a smooth curve. Note that there exists $\delta>0$ such that every $\mathcal{A}=\left(a_{1}, \cdots, a_{n}\right)$-stable curve is also $\mathcal{A}_{\delta}=$ $\left(a_{1}-\delta, \cdots, a_{n}-\delta\right)$-stable. Therefore $\bar{M}_{0, \mathcal{A}}=\bar{M}_{0, \mathcal{A}_{\delta}}$ and $\varphi_{\mathcal{A}}=\varphi_{\mathcal{A}_{\delta}}$, so $\varphi_{\mathcal{A} *}\left(\Delta_{\mathcal{A}_{\delta}}\right)$ is nef by Proposition 3.2. Thus

$$
\begin{aligned}
\varphi_{\mathcal{A} *}\left(\Delta_{\mathcal{A}}\right) & =\varphi_{\mathcal{A} *}\left(K_{\bar{M}_{0, n}}+\sum_{i=1}^{n} a_{i} \psi_{i}\right) \\
& =\varphi_{\mathcal{A} *}\left(K_{\bar{M}_{0, n}}+\sum_{i=1}^{n}\left(a_{i}-\delta\right) \psi_{i}\right)+\delta \varphi_{\mathcal{A} *}(\psi)=\varphi_{\mathcal{A} *}\left(\Delta_{\mathcal{A}_{\delta}}\right)+\delta \varphi_{\mathcal{A} *}(\psi)
\end{aligned}
$$

On $\bar{M}_{0, n}, \psi=\sum_{j=2}^{\lfloor n / 2\rfloor} \frac{j(n-j)}{n-1} D_{j}$ by Lemma 2.9. Thus $\varphi_{\mathcal{A} *}\left(\Delta_{\mathcal{A}}\right)$ is a sum of a nef divisor $\varphi_{\mathcal{A} *}\left(\Delta_{\mathcal{A}_{\delta}}\right)$ and an effective divisor $\delta \varphi_{\mathcal{A} *}(\psi)$ supported on the boundary. Note that $\psi$ and $\varphi_{\mathcal{A} *}(\psi)$ are positive linear combinations of all boundary components. So there exists $\epsilon_{\mathcal{A}, 0}>0$ such that for $P \in N^{1}\left(\bar{M}_{0, \mathcal{A}}\right)$ with $\|P\|_{\mathcal{A}}<\epsilon_{\mathcal{A}, 0}$, $\delta \varphi_{\mathcal{A} *}(\psi)+P$ is an effective sum of boundary divisors. Since $B$ intersects with 
the complement of boundary divisors, $\left(\delta \varphi_{\mathcal{A} *}(\psi)+P\right) \cdot B \geq 0$. Therefore $B$ intersects nonnegatively with $\varphi_{\mathcal{A} *}\left(\Delta_{\mathcal{A}}\right)+P=\varphi_{\mathcal{A} *}\left(\Delta_{\mathcal{A}_{\delta}}\right)+\left(\delta \varphi_{\mathcal{A} *}(\psi)+P\right)$, for every $P$ satisfying $\|P\|_{\mathcal{A}}<\epsilon_{\mathcal{A}, 0}$.

Note that there exist only finitely many strata on $\bar{M}_{0, \mathcal{A}}$. So if we take $\epsilon_{\mathcal{A}}$ to be the minimum among $\epsilon_{\mathcal{A}, 0}, \epsilon_{\mathcal{A}, I}$ and $\epsilon_{\mathcal{A}, J}, \varphi_{\mathcal{A} *}\left(\Delta_{\mathcal{A}}\right)+P$ is nef for all $P \in N^{1}\left(\bar{M}_{0, \mathcal{A}}\right)$ such that $\|P\|_{\mathcal{A}}<\epsilon_{\mathcal{A}}$.

Next, we prove item (2) of Theorem 1.4. In this case, Kapranov's morphisms $\pi_{\mathcal{A}}$ : $\bar{M}_{0, n} \rightarrow\left(\mathbb{P}^{1}\right)^{n} / /{ }_{L} S L(2)$ for any effective ample linearization $L=\mathcal{O}\left(a_{1}, \cdots, a_{n}\right)$ plays the same role as $\varphi_{\mathcal{A}}($ Kap93 $)$.

Theorem 3.6. Assume that $n \geq 5$. Let $\mathcal{A}=\left(a_{1}, \cdots, a_{n}\right)$ be a weight datum satisfying $\sum_{i=1}^{n} a_{i}=2$. Then the log canonical model $\bar{M}_{0, n}\left(K_{\bar{M}_{0, n}}+\sum a_{i} \psi_{i}\right)$ is isomorphic to $\left(\mathbb{P}^{1}\right)^{n} / /{ }_{L} S L(2)$, where $L=\mathcal{O}\left(a_{1}, \cdots, a_{n}\right)$.

Proof. For each subset $I \subset[n]$, set $w_{I}:=\sum_{i \in I} a_{i}$. Let $S$ be the set of $I \subset[n]$ such that

(1) $2 \leq|I| \leq n-2$,

(2) $w_{I}<w_{I^{c}}$ or $w_{I}=w_{I^{c}},|I|<\left|I^{c}\right|$ or $w_{I}=w_{I^{c}},|I|=\left|I^{c}\right|, 1 \in I$.

So there is a bijection between $S$ and the set of irreducible components $D_{I}$ of the boundary divisor of $\bar{M}_{0, n}$. Set $T=\left\{I \subset[n]\left|w_{I} \leq 1,2 \leq\right| I \mid \leq n-2\right\}$ as before. Define

$$
\Delta_{\mathcal{A}}^{\prime}:=(n-4) \sum_{I \in S}\left(-\left(\begin{array}{c}
|I| \\
2
\end{array}\right) \frac{2}{(n-1)(n-2)}+\frac{|I|-1}{n-2} w_{I}\right) D_{I} .
$$

Then by Lemmas 2.8 and 2.9 , it is straightforward to check that $\Delta_{\mathcal{A}}-\Delta_{\mathcal{A}}^{\prime}$ is equal to the right side of (17).

The morphism $\pi_{\mathcal{A}}$ contracts all the boundary divisors except $D_{I}$ with $|I|=2$. The coefficient of $D_{I}$ in $\Delta_{\mathcal{A}}-\Delta_{\mathcal{A}}^{\prime}$ is nonnegative if $|I| \geq 3$ (since $w_{I} \leq 1$ ) and zero if $|I|=2$. Thus $\Delta_{\mathcal{A}}-\Delta_{\mathcal{A}}^{\prime}$ is also effective and supported on the exceptional locus of $\pi_{\mathcal{A}}$. Therefore, by the same argument as in the proof of Theorem 3.1. $\bar{M}_{0, n}\left(\Delta_{\mathcal{A}}\right) \cong \bar{M}_{0, n}\left(\Delta_{\mathcal{A}}^{\prime}\right)$.

For a partition $I_{1} \sqcup I_{2} \sqcup I_{3} \sqcup I_{4}=[n]$, set $w_{j}=w_{I_{j}}=\sum_{i \in I_{j}} a_{i}$. We may assume that $w_{1} \leq w_{2} \leq w_{3} \leq w_{4}$. By Lemma 2.10 it is straightforward to check

$$
\Delta_{\mathcal{A}}^{\prime} \cdot F_{I_{1}, I_{2}, I_{3}, I_{4}}= \begin{cases}0, & w_{4} \geq 1 \\ (n-4)\left(1-w_{4}\right), & w_{4} \leq 1 \text { and } w_{1}+w_{4} \geq 1 \\ (n-4) w_{1}, & w_{4} \leq 1 \text { and } w_{1}+w_{4} \leq 1\end{cases}
$$

These intersection numbers are proportional to those of $\pi_{\mathcal{A}}^{*}\left(\mathcal{O}\left(a_{1}, \cdots, a_{n}\right) / / S L(2)\right)$ in AS08, Lemma 2.2]. Since $N_{1}\left(\bar{M}_{0, n}\right)$ is generated by F-curves, $\Delta_{\mathcal{A}}^{\prime}$ is proportional to the pull-back of the canonical ample divisor $\mathcal{O}\left(a_{1}, \cdots, a_{n}\right) / / S L(2)$ on $\left(\mathbb{P}^{1}\right)^{n} / /{ }_{L} S L(2)$. Therefore $\bar{M}_{0, n}\left(\Delta_{\mathcal{A}}\right) \cong\left(\mathbb{P}^{1}\right)^{n} / /{ }_{L} S L(2)$.

Remark 3.7. Theorem 3.1 shows an interesting relation between log canonical models of stable pointed rational curves parametrized by $\bar{M}_{0, n}$ and that of the parameter space $\bar{M}_{0, n}$. Let $\left(C, x_{1}, \cdots, x_{n}\right)$ be a stable pointed rational curve. Then the $\log$ canonical model $C\left(\omega_{C}+\sum a_{i} x_{i}\right)$ is an $\mathcal{A}$-stable curve. More precisely, it is $\varphi_{\mathcal{A}}\left(C, x_{1}, \cdots, x_{n}\right)$. The same weight datum determines the log canonical model of the parametrized curve $\left(C, x_{1}, \cdots, x_{n}\right)$ and that of the parameter space $\bar{M}_{0, n}$ itself. 
Remark 3.8. Suppose that the weight datum $\mathcal{A}=\left(a_{1}, \cdots, a_{n}\right)$ is symmetric, i.e, $a_{1}=\cdots=a_{n}=\alpha$ for some $2 / n<\alpha \leq 1$. Then by Lemmas 2.8 and 2.9 .

$$
\psi=\sum_{j=2}^{\lfloor n / 2\rfloor} \frac{j(n-j)}{n-1} D_{j}=K_{\bar{M}_{0, n}}+2 D .
$$

So for $\alpha>0$,

$$
K_{\bar{M}_{0, n}}+\alpha \psi=(1+\alpha)\left(K_{\bar{M}_{0, n}}+\frac{2 \alpha}{1+\alpha} D\right) .
$$

Therefore the log canonical model of the pair $\left(\bar{M}_{0, n}, K_{M_{0, n}}+\alpha \psi\right)$ is equal to the log canonical model of the pair $\left(\bar{M}_{0, n}, K_{\bar{M}_{0, n}}+\frac{2 \alpha}{1+\alpha} D\right)$. If we substitute $\beta=\frac{2 \alpha}{1+\alpha}$, then we obtain Theorem 1.2, except the range of bigness of the divisor $K_{\bar{M}_{0, n}}+\beta D$. Hence Theorem 1.4 is a generalization of Simpson's theorem (Theorem 1.2).

Remark 3.9. In [Kee92], Keel proved that

$$
\operatorname{rank} \operatorname{Pic}\left(\bar{M}_{0, n}\right)=\operatorname{dim} N^{1}\left(\bar{M}_{0, n}\right)=2^{n-1}-\left(\begin{array}{l}
n \\
2
\end{array}\right)-1 .
$$

This dimension increases exponentially, so one can expect that the whole picture of the birational geometry of $\bar{M}_{0, n}$ is extremely complicated. The moduli spaces $\bar{M}_{0, \mathcal{A}}$ give a family of birational models for $\bar{M}_{0, n}$. These models are smooth, are the targets of birational morphisms from $\bar{M}_{0, n}$ which are smooth blow-downs, and, most importantly, are moduli spaces for another moduli problem. But from Theorem 3.1] one can conclude that this family of birational models of $\bar{M}_{0, n}$ are detected by only an $n$-dimensional subcone of the effective cone of $\bar{M}_{0, n}$. So it seems that there are still many more birational models of $\bar{M}_{0, n}$ to be discovered.

Remark 3.10. Let $S=\{I \subset[n]|2 \leq| I \mid \leq\lfloor n / 2\rfloor, 1 \in I$ if $|I|=n / 2\}$ be the standard index set of boundary divisors. A divisor $\Delta$ on $\bar{M}_{0, n}$ is called log canonical if

$$
\Delta=r\left(K_{\bar{M}_{0, n}}+\sum_{I \in S} c_{I} D_{I}\right)
$$

for some $r>0$ and $0 \leq c_{I} \leq 1$ (compare to the definition in [AGS10, Definition 6.2]). If $c_{I}=c_{J}$ for $I, J \in S$ such that $|I|=|J|$, then we say $\Delta$ is symmetrically log canonical. For a divisor $\Delta$ which is numerically equivalent to a $\log$ canonical divisor, it is nef if and only if $\Delta$ intersects with F-curves nonnegatively ([FG03, Theorem 4]). So if $\varphi_{\mathcal{A}}^{*} \varphi_{\mathcal{A} *}\left(\Delta_{\mathcal{A}}\right)$ in (6) is $\log$ canonical, computing intersection numbers with $\mathrm{F}$ curves is sufficient to prove Theorem 3.1 .

But in general, $\varphi_{\mathcal{A}}^{*} \varphi_{\mathcal{A} *}\left(\Delta_{\mathcal{A}}\right)$ is not numerically equivalent to a log canonical divisor. For example, if $n=15$ and $\mathcal{A}=(1 / 6,1 / 6, \cdots, 1 / 6)$, then by using the computer algebra system, we can check that $\varphi_{\mathcal{A}}^{*} \varphi_{\mathcal{A} *}\left(\Delta_{\mathcal{A}}\right)$ is not symmetrically log canonical. By Proposition 3.11, it is not numerically equivalent to a log canonical divisor. Thus Theorem 3.1 cannot be proved by checking intersection numbers with F-curves.

Proposition 3.11 is due to the referee.

Proposition 3.11. Let $\Delta$ be an $S_{n}$-invariant divisor on $\bar{M}_{0, n}$. Suppose that $\Delta$ is numerically equivalent to a log canonical divisor. Then $\Delta$ is symmetrically log canonical. 
Proof. By hypothesis, we have

$$
\Delta+\sum a_{i} R_{i}=r\left(K_{\bar{M}_{0, n}}+\sum c_{I} D_{I}\right),
$$

where $R_{i}$ denote the Rulla relations ([Rul06, Lemma 2.1]), $0 \leq c_{I} \leq 1$ for all $I$. The summation is over the set of boundary divisors.

The Rulla relations form an irreducible $S_{n}$-module ([MS11, Proposition 2.3]). Thus $\sum_{\sigma \in S_{n}} \sigma R_{i}=0$ for all $i$. Therefore by symmetrizing both sides of (20), we obtain

$$
\Delta=r\left(K_{\bar{M}_{0, n}}+\sum_{j=2}^{\lfloor n / 2\rfloor} \frac{1}{\left(\begin{array}{c}
n \\
j
\end{array}\right)}\left(\sum_{|I|=j} c_{I}\right) D_{j}\right) .
$$

Since $0 \leq c_{I} \leq 1$, we have $0 \leq \frac{1}{\left(\begin{array}{c}n \\ j\end{array}\right)}\left(\sum_{|I|=j} c_{I}\right) \leq 1$; hence $\Delta$ is symmetrically log canonical.

\section{ACKNOWLEDGEMENTS}

It is a great pleasure for the author to thank his advisor, Young-Hoon Kiem. Originally, finding a universal formula for $\log$ canonical models of $\bar{M}_{0, n}$ was a question raised by him. Without his patience and advice, it would have been impossible for the author to finish this project. The author would also like to thank Maksym Fedorchuk and the referee for invaluable comments and suggestions.

\section{REFERENCES}

[AGS10] V. Alexeev, A. Gibney and D. Swinarski. Conformal blocks divisors on $\bar{M}_{0, n}$ from sl 2 . arXiv:1011.6659.

[AS08] V. Alexeev and D. Swinarski. Nef divisors on $\bar{M}_{0, n}$ from GIT. arXiv:0812.0778.

[AC96] E. Arbarello and M. Cornalba. Combinatorial and algebro-geometric cohomology classes on the moduli spaces of curves. J. Algebraic Geom. 5 (1996), no. 4, 705-749. MR 1486986 (99c:14033)

[AC99] E. Arbarello and M. Cornalba. Calculating cohomology groups of moduli spaces of curves via algebraic geometry. Inst. Hautes Études Sci. Publ. Math. No. 88 (1998), 97-127 (1999). MR1733327 (2001h:14030)

[Deb01] O. Debarre. Higher-dimensional algebraic geometry. Universitext, Springer-Verlag, New York, 2001. MR.1841091 (2002g:14001)

[FG03] G. Farkas and A. Gibney. The Mori cones of moduli spaces of pointed curves of small genus. Trans. Amer. Math. Soc. 355 (2003), no. 3, 1183-1199. MR.1938752 (2003m:14043)

[Fed10] M. Fedorchuk. Moduli spaces of weighted stable curves and log canonical models of $\bar{M}_{g, n}$. Math. Res. Lett. 18 (2011), no. 4. MR2831833

[FS08] M. Fedorchuk and D. Smyth. Ample divisors on moduli spaces of pointed rational curves. J. Algebraic Geom. 20 (2011), no. 4, 599-629. MR2819671

[GG11] N. Giansiracusa and A. Gibney. The cone of type A, level one conformal blocks divisors. arXiv:1105.3139.

[GS10] N. Giansiracusa and M. Simpson. GIT compactifications of $\bar{M}_{0, n}$ from conics. Inter. Math. Res. Notices 2011, no. 14, 3315-3334. MR2817681(2012j:14042)

[GKM02] A. Gibney, S. Keel and I. Morrison. Toward the ample cone of $\bar{M}_{g, n}$. J. Amer. Math. Soc. 15 (2002), no. 2, 273-294. MR.1887636 (2003c:14029)

[HM98] J. Harris and I. Morrison. Moduli of curves. Graduate Texts in Mathematics, 187. Springer-Verlag, New York, 1998. MR 1631825 (99g:14031)

[Has03] B. Hassett. Moduli spaces of weighted pointed stable curves. Adv. Math. 173 (2003), no. 2, 316-352. MR.1957831 (2004b:14040)

[HK00] Y. Hu and S. Keel. Mori dream spaces and GIT. Michigan Math. J. 48 (2000), 331-348. MR 1786494 (2001i:14059) 
[Kap93] M. Kapranov. Chow quotients of Grassmannians. I. I. M. Gelfand Seminar, 29-110, Adv. Soviet Math., 16, Part 2, Amer. Math. Soc., Providence, RI, 1993. MR.1237834 (95g:14053)

[Kee92] S. Keel. Intersection theory of moduli space of stable n-pointed curves of genus zero. Trans. Amer. Math. Soc. 330 (1992), no. 2, 545-574. MR.1034665 (92f:14003)

[KMc96] S. Keel and J. McKernan. Contractible extremal rays on $M_{0, n}$. arXiv:9607009.

[KM11] Y.-H. Kiem and H.-B. Moon. Moduli spaces of weighted stable pointed rational curves via GIT. Osaka J. Math. 48 (2011), no. 4, 1115-1140. MR2871297

[Kol90] J. Kollár. Projectivity of complete moduli. J. Differential Geom. 32 (1990), no. 1, 235268. MR 1064874 (92e:14008)

[Knu83] F. Knudsen. The projectivity of the moduli space of stable curves. II. The stacks $M_{g, n}$. Math. Scand. 52 (1983), no. 2, 161-199. MR702953 (85d:14038a)

[Mo11] H.-B. Moon. Birational geometry of moduli spaces of curves of genus zero. Ph.D. dissertation, Seoul National University, 2011.

[MS11] I. Morrison and D. Swinarski. The $S_{n}$-module structure of $\operatorname{Pic}\left(\bar{M}_{0, n}\right)$, preprint.

[Mum77] D. Mumford. Stability of projective varieties. Enseignement Math. (2) 23 (1977), no. 12, 39-110. MR0450272(56:8568)

[Pan97] R. Pandharipande. The canonical class of $\bar{M}_{0, n}\left(\mathbb{P}^{r}, d\right)$. Internat. Math. Res. Notices 1997, no. 4, 173-186. MR.1436774 (98h:14067)

[Rul06] W. Rulla. Effective cones of quotients of moduli spaces of stable n-pointed curves of genus zero. Trans. Amer. Math. Soc. 358 (2006), no. 7, 3219-3237. MR2216265 (2007b:14053)

[Sim07] M. Simpson. On log canonical models of the moduli space of stable pointed curves. arXiv:0709.4037.

Department of Mathematics, University of Georgia, Athens, Georgia 30602

E-mail address: hbmoon@math.uga.edu 